(REVIEW ARTICLE)

\title{
Therapeutic potential of hedgehog signaling pathway agonist
}

\author{
Kumari Poonam, Sharma Ankita, Singla Shivali * and Goyal Sachin \\ Himalayan Institute of Pharmacy, Kala-Amb, Sirmour (H.P.), India. \\ Publication history: Received on 23 January 2019; revised on 10 June 2019; accepted on 14 June 2019
}

Article DOI: https://doi.org/10.30574/wjarr.2019.2.2.0014

\begin{abstract}
The Hedgehog (Hh) signaling pathway plays an important role in the growth, development, and homeostasis of many tissues in vertebrates and invertebrates in embryonic stage. However, Hh signaling is also involved in postnatal processes such as tissue repair and adult immune responses. To some extent, Hh signaling has also been shown to be a target for some pathogens like HIV, EVB and influenza that presumably utilize the pathway to control the local infected environment. In present review, we discuss the detailed mechanism of the Hedgehog (Hh) signaling pathway involved in various disease being a target. We also give a range of agonist and on the possibility of using small molecule modulators of Hh signaling as effective therapies for a wider range of human diseases.
\end{abstract}

Keywords: Hedgehog (Hh); Smoothened (Smo); Agonist; Cancer; Autism

\section{Introduction}

Hedgehog $(\mathrm{Hh})$ is an intercellular signaling molecule first observed in fruit flies of the genus Drosophila. It is a highly conserved developmental pathway involved in organogenesis, stem cell maintenance, and tissue repair/regeneration [1]. Hedgehog protein is a paracrine factor which enhances cell proliferation and differentiation in many cell types [2]. One of the key differences between flies and mammals is the redundancy in pathway components. Whereas Drosophila has only one Hh ligand and one primary receptor, mammals have three ligands (Sonic Hedgehog-Shh, Indian HedgehogIhh, and Desert Hedgehog-Dhh) and two primary receptors (Ptch1 and Ptch2). Similarly, Drosophila has only one transcriptional effector, Ci, which can act as both an activator and repressor, whereas mammals have three, Gli1, Gli2, and Gli3, where Gli2 is the primary activator, Gli3 is the primary repressor, and Gli1 is a target gene that acts as an activator in a positive feedback loop. There are also differences in how the message is relayed from Smo to downstream components. Namely, mammalian cells require intracellular transport components housed in primary cilia; this subcellular compartmentalization is not required in Drosophila. Regardless of these differences, the method by which canonical Hh signals as a morphogen in all systems is understood to be by creating precise balances between repressor and activator forms of $\mathrm{Gli} / \mathrm{Ci}$, which activate target genes with varying numbers and affinities of transcription factor binding sites. One of the key The Hh family consist of secreted proteins or ligands, Sonic Hedgehog (Shh), Indian Hedgehog (Ihh), and Desert Hedgehog (Dhh) of which Shh is the most studied ligand [3]. Function of Shh is to regulate the growth of neuronal, CNS tissue development. Ihh is to regulate bone and cartilage development. Dhh is essential for germ cell development in the testis and peripheral nerve sheath formation [4].

Additionally, in the normal adult physiology this pathway also participates in stem cell maintenance, tissue repair and regeneration [5]. In adults, the Hh pathway remains active and is involved in regulation of tissue homeostasis, continuous renewal and repair of adult tissues, and stem cell maintenance [6]. Hh signaling pathway is controlled by membrane proteins Patched (Ptch) and Smoothened (Smo), the signal transducer (the effector component), transmembrane receptors (Ptch1-2), downstream regulators (SuFu) and as well as transcription factors (Gli1-3) [7]. When there is no ligand bound to Ptch, Ptch inhibits Smo and keeps the downstream pathway inactivated (Figure: 1) When Hh ligand binds to Ptch, the inhibition of Ptch on Smo is relieved and the Hh-signaling pathway is activated. One

\footnotetext{
${ }^{*}$ Corresponding author

E-mail address: singlashivali@gmail.com
} 
of the broadly accepted mechanisms of Hh-signaling target genes response is through the binding of Gli family transcription factors to Gli-binding sites in the regulatory sequence of Hh regulated genes [8].

Abnormal activation of the Hh signaling pathway has been linked to several types of human cancers including pancreatic cancer, prostate cancer, colon cancer, basal cell carcinoma and medulloblastoma, and the development of small molecule inhibitors of this pathway represents a promising route toward novel anticancer therapeutics [9].

\section{Hedgehog Mechanism}

There are two proteins in surface of cell that is Patch and Smoothen. Hh signaling pathway is controlled by membrane proteins Patched (Ptch) and Smo. Smoothened (Smo) the signal transducer (the effector component), transmembrane receptors (Ptch1-2), downstream regulators (SuFu) and as well as transcription factors (Gli1-3) [10].

A Hh pathway OFF

B Hh pathway ON

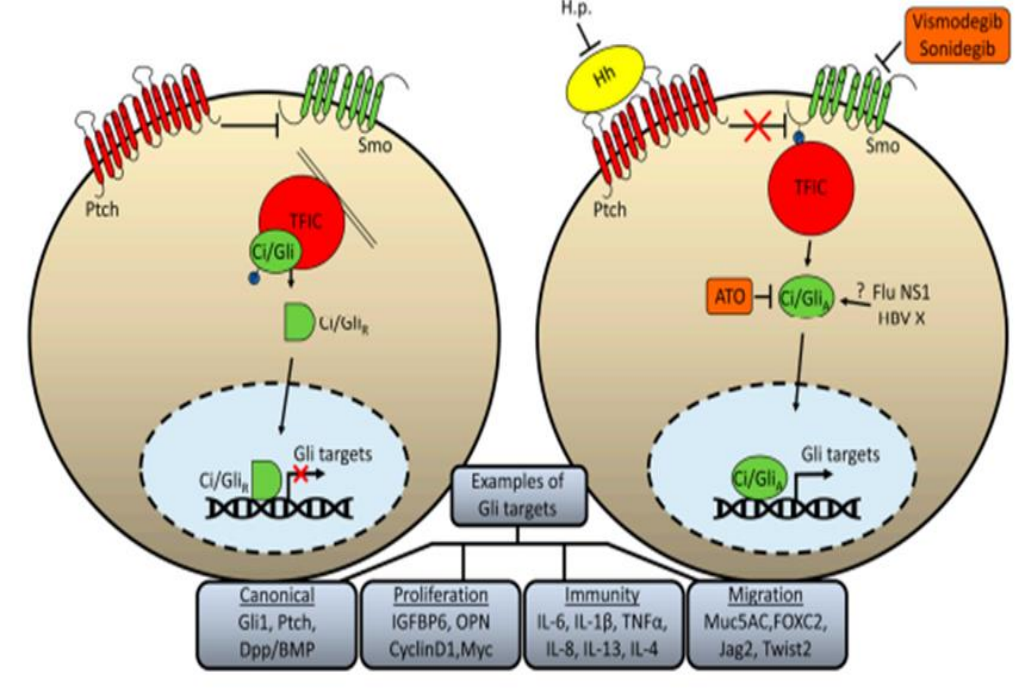

Figure 1 Mechanism of Hh Pathway (A) In the absence of Hh ligands (B) Interaction of Hh ligands [11]

\subsection{Activating Hh Signaling Through the GPCR-Like SMO Receptor}

As a receptor class, GPCRs are considered excellent drugtargets because they are often regulated through interactions with small natural ligands [12]. Specifically, studies of classic GPCRs, such as the adrenergic receptors, show thatin the absence of endogenous ligand (agonists) these receptor works in multiple inter convertible conformations that are predominately inactive $[13,14]$. Upon exposure to theirnatural ligands, however, the active receptor forms are preferentiallystabilized, allowing them to readily engageG-protein couplers and to create signaling-competent complexes. Multiple compound classes have been isolated onthe basis of their ability to compete for the binding ofadrenergic receptors by their natural ligands. These competitorscan mimic the natural ligand activity (agonists) orinterfere with it (antagonists).

In addition to the binding sites for natural or endogenous agonists (orthosteric sites), many GPCRs have also beenfound to have allosteric sites. These sites can bindnatural ligands, as in the case of $\mathrm{Zn}$ ions and heparin forthe dopamine and neurokinin receptors, respectively, orbind synthetic drugs such gallamine, in the case of themuscarinic receptors [15]. Binding of small molecules tothese allosteric sites can modulate activity of a receptorwithout directly mimicking or competing out the interactionof ligands to the orthosteric sites. In summary, GPCRshave an array of potential regulatory binding sites, orpotential drug targets [16].

\subsection{Activation of the Hedgehog Pathway in Human Cancer}

$\mathrm{Hh}$ signaling in human cancers was achieved by the discovery that mutations of the human homolog of the Drosophilapatched gene (PTCH1) are associated with a rare hereditary form of BCC (basal cell nevus syndrome, also called Gorlin syndrome). Gorlin syndrome is a rare autosomal genetic disorder with two distinct sets of phenotypes: a high risk of BCC and a predisposition to develop medulloblastoma and developmental defects such as bifid ribs and ectopic calcification. BCC one ofthe most common human cancers, regularly shows abnormalities of the Hh pathway 
arising from mutations in PTCH1 (50\%), SMO (10\%) and other genes, including SuFu. About one third of medulloblastomas show activated Hh signaling, and mutations influencing the signaling cascade could be commonly detected within the PTCH1 gene. Increasing evidence supports the notion that the activation of Hh signaling plays an important pathophysiolocial role in many types of human cancer. It is postulated that over $30 \%$ of human cancers are presented with activated Hh signaling, including brain tumors, melanomas, leukemias, lymphomas, gastrointestinal, kidney, bladder, pancreatic, liver, prostate, lung, ovarian and breast cancers. In those cancers, gene mutation is not primarily responsible for activated Hh signaling, but rather caused by ligand-dependent mechanisms or non-canonical Hh signaling activation [17].

\subsection{The Role of Hedgehog Signaling in Cancer Initiation and Progression}

Hh signaling is involved in different stages of carcinogenesis. Hh signaling plays a significant role in the initiation of esophageal adenocarcinomas. Furthermore, activation of this pathway was observed in pancreatic intraepithelial neoplasia (PanIN) lesions as well in metastases of pancreatic cancer, suggesting that Hh signaling is also important for pancreatic cancer.

Hh signaling plays an important role in the development and progression of glioma, breast cancer, ovarian cancer, leukemia, and B-cell lymphoma. The modes of Hh signaling in cancer development may vary from one tumor type to another. It is noteworthy that correlation of Hh target gene expression with tumor specimens is not sufficient to clarify the role of $\mathrm{Hh}$ signaling in a specific cancer type. Establishing animal models using tissue specific activation of Hh signaling is crucial for understanding the role of Hh signaling in tumor progression [17].

\section{Therapeutic Potential of Hh-Pathway Agonist}

Various studies in mammals have shown that Hh genes are expressed in discrete areas of the adult organism and may function in the normal maintenance of mature organ systems. In addition, the regenerative healing of vascular and skeletal tissues following acute injuries appearsto be aided by re-activating the Hh-signaling cascade. Hh pathway may represent a point of intervention for treatingcertain degenerative disorders. Two recent studies in models of Parkinson's disease and peripheral nerve damagesupport this claim, by demonstrating that pathway activation with an Hh-protein ligand has therapeutic value. On the basis of current understanding of these models and the specific mechanism of action of the $\mathrm{Hh}$ agonists, predict that an agonist-derivative with low toxicity and favorable pharmacokinetics would replicate these positive results. As a drug, an Hh agonist would represent an attractive alternative to an expensive Hh-protein therapeutic [19].

The pivotal role of Hh signaling in stem cells, during development of many tissues and organs, and in remodeling, repair, and regeneration of adult tissues, prompted to search small molecules that modulate the Hh pathway. While Hh inhibition has found application in anticancer therapy, the activation of the Hh pathway may promote tissue regeneration and repair after severe injury in numerous organs. Small molecules that act as positive modulators of Smo are able to promote or improve human embryonic stem cell differentiation and have been proposed as therapeutic tools for stimulating issue functions or restoring repair mechanisms that are compromised by disease. Smo agonists have been also used to facilitate tissue and organ repair, and for implantation of engineered tissues. Of particular importance is the osteo inductive activity of Smo agonists which are able to increase bone mass, thus representing attractive molecules in the field of osteopenia, osteoporosis and bone diseases. Hh agonists act also as an effective tool for bone formation after forearm amputation in mouse, and promote proliferation and survival of cortical/hippocampal cells, without affecting their differentiation or inducing apoptosis [20].

\subsection{Hedgehog Pathway in Neurological Disorders}

Hh signaling has been identified as an integral component for neural system patterning in both the central and peripheral nervous systems (CNS and PNS). In the CNS, Shh signaling regulates the specification of multiple neural cell types, including motor, dopaminergic, and basal forebrain cholinergic neurons. Neurological disorders such as tumor induced injury to surrounding brain and cause Shh activity in gliomas. In most types of brain injury including brain tumors reactive astrocytes are present. Therefore, Shh pathway activation in reactive astrocytes occurs during nonneoplastic brain injury. The expression of Shh in reactive astrocytes may play a role in promoting cell proliferation during brain injury. This is analogous to its role during certain types of brain tumors [21].

Shh stimulates proliferation of neural precursors. Dhh appears to play an essential role in PNS development. In addition to its role during neural system development, proper Hh signaling plays a crucial role in the adult nervous system; most notably, through regulation of neuronal stem cell populations and neuronal cell fate determination. Hh signaling as a 
therapeutic strategy against CNS neurodegenerative disorders has focused on Parkinson's disease (PD), a degenerative disorder characterized by the loss of dopaminergic neurons in the substantia nigra.

Shh has also demonstrated neuroprotective effects in an in vitro model of amyotrophic lateral sclerosis following oxidative insult. Induction of $\mathrm{Hh}$ signaling has been studied as a potential treatment for peripheral diabetic neuropathy and nerve injury. These studies indicate that up regulation of Hh signaling could promote survival, regeneration, or increased function of remaining neurons and could be a valid strategy for treating numerous neurodegenerative disorders [22].

\subsubsection{Hedgehog Pathway in Autism Disorders}

Autism disorders are common neurodevelopmental disorders and associated with impairments in social interactions, communication and behavior [23]. Shh signaling plays a neuro protective role against oxidative stress in autism. Sonic hedgehog signaling and brain-derived neurotrophic factor play a neuro-protective role against oxidative stress in autism. Sonic hedgehog also increases Bcl-2 expression and the activities of superoxide dismutase and glutathione peroxidase. The level or activity of Bcl-2, brain derived neurotrophic factor, and the activities of superoxide dismutase and glutathione peroxidase are decreased in autism. Sonic hedgehog also decreases the production of malondialdehyde that its level is high in autism. Therefore, it is supposed that sonic hedgehogmay be associated with oxidative stress in autism through other pathways too. The activation of sonic hedgehog (Shh) signaling protects neurons from apoptosis induced by oxidative stress. Shh enhances SOD and GSH-PX activities. It also lowers Malondialdehyde production. Shh activation enhances Bcl-2 expression and reduces the expression of Bax SHH activation. It increases the BDNF level too. Oxidative stress may explain some symptoms in autism and it is a hypothesized target for the treatment of autism [24].

\subsection{Diabetes, obesity and Ischemia}

Hh signaling to regulate metabolism demonstrated a conserved role in Drosophila and mammals in which Hh signaling inhibited fat formation. A genome-wide RNAi screen in adult Drosophila, designed to identify genes linked to obesity, identified the Hh signaling pathway as the top scoring pathway specific to the formation of fat. Interestingly, this study demonstrated that the pathway specifically blocks white adipogenesis, but has no effect on brown adipogenesis. A SmoCa2+-Ampk axis, active in the primary cilia, rapidly induces Warburg-like metabolic reprogramming that is required for proper metabolic function. Activation of this axis in vivo induces insulin-dependent glucose uptake in both muscle and brown fat tissue, suggesting that Hh pathway agonists hold therapeutic potential for the treatment of diabetes and obesity [25].

Ischemic diseases are characterized by reduced blood flow to the tissues, resulting in a decrease in oxygen, glucose, and other nutrients required for proper cellular function. The most prominent forms of ischemia include ischemic heart disease, myocardial infarction, and stroke. A primary therapeutic strategy to treat ischemia is revascularization of the damaged or degenerative blood vessels. The Hh signaling pathway is integral during the development of the cardiovascular system and is sufficient to promote the formation of new coronary vessels in adults [26].

\subsection{Hedgehog Pathway in tissue damage and repair}

After the development process completion, the level of Hh ligand expression, Ptch, Smo and Gli1 declines to low levels in normal healthy tissues. In adults, the Hh pathway remains active and is involved in regulation of tissue homeostasis, continuous renewal and repair of adult tissues, and stem cell maintenance. Hh signaling is aberrantly activated in Osteoarthritis (OA) cartilage and promotes the hypertrophic phenotype of chondrocytes. The inhibition of Hh signaling reduces disease severity in OA animal models, act as therapeutic target for OA treatment. Shh and Ihh pathway was expressed by chondrocytes in response to mechanical stress. In the recent study, Ihh gene transfer induces chondrogenic differentiation of mesenchymal stem cells and the ability of Shh to induce redifferentiation of dedifferentiated chondrocytes by the stimulation of SOX-9, BMP-2 and IGF expression was reported (Mariani et al., 2014). In diabetic state, through the activation of Shh and induction of NO release, endothelial cells protected against injury and improves delayed wound healing. Shh proteins have a productive effect on cardiac injuries via recruitment of endothelial progenitor cells and fibroblast proliferation [27].

\subsection{Hedgehog Pathway in Osteo degenerative disorders}

Hh signaling is essential for guiding the proper proliferation and differentiation of osteo progenitor cells during embryonic bone formation; activity that is primarily linked to Ihh. These effects can either be through direct initiation of osteogenic differentiation in osteoblast progenitor cells or via an indirect mechanism in which Ihh induces expression of other cellular factors, primarily, parathyroid hormone-related protein (PTHrP) that initiate bone formation. Selective 
upregulation of Hh signaling in mature osteoblasts led to excessive bone resorption and severe osteopenia, a precursor state to osteoporosis. These data suggest that activation of Hh signaling has the potential to generate new bone growth and may hold potential as a treatment for degenerative bone diseases such as osteoporosis; however, detrimental side effects, including increased risk for osteoporosis, are possible with prolonged pathway activation.

\subsection{Hedgehog Pathway in Hair growth}

Shh plays a vital role in hair follicle morphogenesis during normal skin development and continues an essential role in regulating follicular growth and cycling in adults. While not essential for hair follicle initiation during embryonic development, Shh is required for controlling growth and morphogenesis of the follicle past the hair germ stage. The reduction in hair follicle papillae correlated with decreased expression of both Gli1 and Ptch1. In adults, Shh mediates the transition from the resting to the growth stage during the normal follicle cycle and anti-Shh antibodies prevented hair growth in adult mice. These studies suggest activation of Hh signaling holds therapeutic potential in diseases characterized by decreased follicular proliferation and altered cycling.

\subsection{Hedgehog Pathway in neuro epithelial cell proliferation}

Sonic Hedgehog/Gli signaling pathway play critical role in expansion and modelling of chick optic tectum. This effect is due to the mitosis that increases the neuro epithelial cell proliferation and modulates spatial organization of neuro epithelial cell proliferation. Hh pathway plays important role in cell differentiation and proliferation, therefore by using its small molecule modulators, revival from neuro degeneration can be hypothesized [28].

\section{Conclusion}

The Hedgehog (Hh) signaling pathway plays an integral role incorrect tissue patterning of a variety of different cell types during embryonic development. There are three Hh proteins Sonic (Shh), Indian (Ihh), and Desert (Dhh) that serve as ligands to initiate pathway signaling. Recent years have seen an increase in describing the regenerative role of the $\mathrm{Hh}$ pathway in the central and peripheral nervous systems, the process of bone formation, and the vasculature.Multiple research groups have demonstrated that activation of Hh signaling has potential therapeutic applications in a variety of disease states, including neurodegenerative and osteo degenerative disorders, diabetes, and ischemia.To date, each compound identified as an Hh agonist has been shown to exert its effects through direct binding and modulation of Smo, a key regulator of Hh signaling and the most "druggable" target within the pathway.

\section{Compliance with ethical standards}

\section{Acknowledgments}

The author' s truthfully admit the contribution and technical assistance of Himalayan Institute of Pharmacy, HGPI, Kala-Amb, Sirmour (H.P.) for assistance during review process of the manuscript.

\section{Disclosure of conflict of interest}

There is no conflict of interest.

\section{References}

[1] Abidi A. (2014). Hedgehog signaling pathway: a novel target for cancer therapy: vismodegib, a promising therapeutic option in treatment of basal cell carcinomas. Indian journal of pharmacology, 46(1), 3.

[2] Elia D, Madhala D, Ardon E, Reshef R and Halevy O. (2007). Sonic hedgehog promotes proliferation and differentiation of adult muscle cells: Involvement of MAPK/ERK and PI3K/Akt pathways. Biochimica et Biophysica Acta (BBA)-Molecular Cell Research, 1773(9), 1438-46.

[3] Amankulor NM, Hambardzumyan D, Pyonteck SM, Becher OJ, Joyce JA and Holland EC. (2009). Sonic hedgehog pathway activation is induced by acute brain injury and regulated by injury-related inflammation. Journal of Neuroscience, 29(33), 10299-308.

[4] Hui CC and Angers S. (2011). Gli proteins in development and disease. Annual review of cell and developmental biology, 27, 513-37. 
[5] Kar S, Deb M, Sengupta D, Shilpi A, Bhutia SK and Patra SK. (2012). Intricacies of hedgehog signaling pathways: a perspective in tumorigenesis. Experimental cell research, 318(16), 1959-72.

[6] Gupta S. (2010). Review: Trageting the Hedgehog pathway in cancer. Therapeutic Advances in Medical Oncology, 2(4), 237-250.

[7] Manetti F, Taddei M and Petricci E. (2014). Structure-activity relationships and mechanism of action of small molecule smoothened modulators discovered by high-throughput screening and rational design. In the Smoothened Receptor in Cancer and Regenerative Medicine. Springe International Publishing, 43-107.

[8] Lu Y, Li J, Cheng J and Lubahn DB. (2015). Genes targeted by the Hedgehog-signaling pathway can be regulated by Estrogen related receptor $\beta$. BMC molecular biology, 1, 16.1-16.4.

[9] Ohashi T, Tanaka Y, Shiokawa Z, Banno H, Tanaka T, Shibata S, Satoh Y, Yamakawa H, Yamamoto Y, Hattori H and Kondo S.. (2015). Synthesis and evaluation of hedgehog signaling inhibitor with novel core system. Bioorganic \& medicinal chemistry, 23(15), 4777-4791.

[10] Manetti F, Petricci E, Gabrielli A, Mann A, Faure H, Gorojankina T, Brasseur L, Hoch L , Ruat M and Taddei M. (2016). Design, synthesis and biological characterization of a new class of osteogenic (1H)-quinolone derivatives." European Journal of Medicinal Chemistry, 121, 747-757.

[11] Haixia L, Jinghua L and Limin F. (2015) Hedgehog signaling pathway as a therapeutic target for ovarian cancer. Cancer epidemiology, 40, 152-157.

[12] Howard AD, McAllister G, Feighner SD, Liu Q, Nargund RP, Van der Ploeg LH and Patchett AA. (2001). Orphan Gprotein-coupled receptors and natural ligand discovery. Trends Pharmacol Sci, 22, 132-140.

[13] Civelli O, Nothacker HP, Saito Y, Wang Z, Lin SH and Reinscheid RK, (2001). Novel neurotransmitters as natural ligands of orphan Gprotein-coupled receptors. Trends Neuroscience, 24, 230-237.

[14] Dohlman HG, Thorner J, Caron MG and Lefkowitz R. (1991). Model systems for the study of seventransmembrane-segment receptors. Annu Rev Biochem, 60, 653-688.

[15] Christopoulos A. (2002). Allosteric binding sites on cell-surface receptors: novel targets for drug discovery. Nat Rev Drug Disc, 1, 198-210.

[16] Taipale J, Cooper MK, Maiti T and Beachy PA. (2002). Patched acts catalytically to suppress the activity of Smoothened. Nature, 418, 892-897.

[17] Szkandera J, Kiesslich T, Haybaeck J, Gerger A and Pichler. (2013). Hedgehog signaling pathway in ovarian cancer." International journal of molecular sciences.14 (1), 1179-1196.

[18] Hadden, MK. (2014). Hedgehog Pathway Agonism: Therapeutic Potential and Small-Molecule Development. Chem Med Chem, 9.1, 27-37.

[19] Amankulor NM, Hambardzumyan D, Pyonteck SM, Becher OJ, Joyce JA and Holland EC. (2009). Sonic hedgehog pathway activation is induced by acute brain injury and regulated by injury-related inflammation. Neurobiology of Disease, 29(33), 10299-10308.

[20] Gupta S, Takebe N and LoRusso P. (2010) Review: Targeting the Hedgehog pathway in cancer. Therapeutic Advances in Medical Oncology, 2(4), 237-250.

[21] Mariani E, Pulsatelli L and Facchini A. (2014). Signaling pathways in cartilage repair. International Journal of Molecular Sciences, 15(5), 8667-8698.

[22] Monireh Dashty. (2014). Hedgehog signaling pathway is linked with age related diseases. Journal of Diabetes and Metabolism, 5, 350 .

[23] Caronna, EB, Milunsky, JM and Tager-Flusberg, H. (2008). Autism spectrum disorders: clinical and research frontiers. Arch. Dis. Child, 93, 518-523.

[24] Ghanizadeh A. (2012). Malondialdehyde, Bcl-2, superoxide dismutase and glutathione peroxidase may mediate the association of sonic hedgehog protein and oxidative stress in autism. Neurochemical research, 37.4, 899-901.

[25] Hadden MK. (2014). Hedgehog Pathway Agonism: Therapeutic Potential and Small-Molecule Development. Chem Med Chem, 9.1, 27-37.

[26] Bijlsma MF and Spek CA. (2010). The Hedgehog morphogen in myocardial ischemia-reperfusion injury. Exp. Biol. Med. 235, $447-454$. 
[27] Mak KK, Bi Y, Wan C, Chuang PT, Clemens T, Young M and Yang Y. (2008). Hedgehog signaling in mature osteoblasts regulates bone formation and resorption by controlling PTHrP and RANKL expression. Dev Cell, $14(5), 674-88$

[28] Wang LC, Liu ZY, Gambardella L, Delacour A, Shapiro R, Yang J, Sizing I, Rayhorn P, Garber EA, Benjamin CD, Williams KP, Taylor FR, Barrandon Y, Ling L and Burkly LC. (2000). Regular articles: conditional disruption of hedgehog signaling pathway defines its critical role in hair development and regeneration. J Invest Dermatol, 114, 901-908.

\section{How to cite this article}

Kumari P, Sharma A, Singla S and Goyal S. (2019). Therapeutic potential of hedgehog signaling pathway agonist. World Journal of Advanced Research and Reviews, 2(2), 10-16. 